\title{
32. INVESTIGATION OF THE MOTION OF PERIODIC COMET GIACOBINI-ZINNER AND THE ORIGIN OF THE DRACONID METEOR SHOWERS OF 1926, 1933 AND 1946
}

\author{
YU. V. EVDOKIMOV \\ Kazan University, Kazan, U.S.S.R.
}

\begin{abstract}
Orbital elements of P/Giacobini-Zinner have been obtained from 577 observations at the eight apparitions 1900 to 1965 by linking apparitions in pairs. By this technique we established that the comet has a nongravitational secular deceleration amounting, on the average, to 0.081 day $/(\text { period })^{2}$. As a result of the comet's approach to Jupiter in 1969, a return of the Draconid meteor shower is possible on 1972 October $8^{\mathrm{d}} 15^{\mathrm{h}} 45^{\mathrm{m}}$ UT. The 1946 meteor shower was due to meteoroids ejected forward along the orbit of the comet in 1940 with velocities of $14 \mathrm{~m} \mathrm{~s}^{-1}$. The meteor showers of 1933 and 1926 were apparently produced by meteoroids ejected in 1900 with velocities of $15.0 \mathrm{~m} \mathrm{~s}^{-1}$ forward and $14.5 \mathrm{~m} \mathrm{~s}^{-1}$ backward, respectively.
\end{abstract}

Because of its association with the very strong meteor showers of 1933 and 1946 periodic comet Giacobini-Zinner is of particular interest. The comet was first discovered in 1900 by Giacobini, and having a period of approximately $6.5 \mathrm{yr}$, it was observed also in 1913,1926, 1933,1939, 1946, 1959, and 1965; it was missed at its returns in 1907, 1920, and 1953.

A total of 577 observations, made at 47 observatories, have been reported. Attempts to link successive apparitions (Evdokimov, 1963) convinced us that the motion of this comet is not governed by pure gravitational theory. The elements of the orbit vary irregularly, and it is impossible to represent with reasonable accuracy all these changes for the whole interval 1900 to 1965.

The same conclusion was made by Dubyago in the case of $\mathrm{P} /$ Brooks 2 . In his last work (Dubyago, 1956) he abandoned his method for determining variations in the mean daily motion and eccentricity from equations. Instead, he calculated orbital elements from each pair of successive apparitions and then compared, for some osculation epoch during each apparition, the elements obtained by linking that apparition with the preceding one and with the following one. This technique roughly simulates, in the form of a jump, the accumulated nongravitational change in the elements over a comparatively small arc near perihelion.

We have made use of the same technique in this investigation of the motion of P/Giacobini-Zinner. First we linked the four apparitions 1926-46 in pairs, with the result

Epoch and osculation 1933 July 27.0 ET

1926-1933

$$
\begin{array}{rlrl}
M_{0} & =1.77099 & \pm 0.00011 \\
\omega & =171.77039 & & \pm 0.00070 \\
\Omega & =196.24331 & & \pm 0.00050 \\
i & =30.68403 & \pm 0.00040 \\
e & =0.7159891 & \pm 0.0000004 \\
a & =3.5193712 & \pm 0.0000030
\end{array}
$$

$1933-1939$

$\left.\begin{array}{rl}1.76944 & \pm 0.00006 \\ 171.76983 & \pm 0.00026 \\ 196.24323 & \pm 0.00046 \\ 30.68393 & \pm 0.00017\end{array}\right\} \quad 1950.0$


Epoch and osculation 1939 October 14.0 ET

\begin{tabular}{|c|c|c|c|c|}
\hline \multicolumn{2}{|c|}{ 1933-1939 } & \multicolumn{3}{|c|}{ 1939-1946 } \\
\hline$M_{0}=341.11596$ & \pm 0.00007 & 341.11575 & \pm 0.00032 & \\
\hline$\omega=171.79101$ & \pm 0.00026 & 171.79169 & \pm 0.00025 & \\
\hline$\Omega=196.24993$ & \pm 0.00046 & 196.24946 & $\pm 0.00020\}$ & 1950.0 \\
\hline$i=30.74130$ & \pm 0.00017 & 30.74123 & \pm 0.00013 & \\
\hline 0.7166606 & \pm 0.0000007 & 0.7166605 & \pm 0.0000003 & \\
\hline 3.5138522 & \pm 0.0000016 & 3.5139385 & \pm 0.0000019 & AU. \\
\hline
\end{tabular}

Of the total of 173 observations made at these four apparitions, 30 were erroneous or of low precision and therefore rejected. The remainder were combined into normal places. The mean errors corresponding to the three orbits were \pm 1 ".85, \pm 0 ".75 and \pm 1 ".42 respectively, and the maximum residuals were about $\pm 2 " .5, \pm 1$. 5 and $\pm 3^{\prime \prime}$.

Integration of the 1926-1933 orbit back to 1913 gave residuals of $1200^{\prime \prime}$, while integration of the 1939-1946 orbit forward to 1959 gave residuals of 1400".

The differences between the above pairs of elements are listed in Table $I$ in the columns headed 1933 and 1939, respectively, and also designated (a) and (b). Column (c) is essentially a mean, and these variations were applied to the 1926 elements before integrating back to 1920. The elements so obtained for an epoch near perihelion in 1920 were corrected using the same mean variations and the integration then continued to 1913. The resulting $(\mathrm{O}-\mathrm{C})$ residuals in 1913 were of the order 2 " to 20", suggesting that the mean variations of the elements conform to reality.

After an orbit improvement using the 1913 observations we obtained the following comparison of the elements for an epoch in 1926. The mean errors are \pm 2 ".42 and \pm 2 ". 30 , respectively, and the differences are given in Table I as column (d).

Epoch and osculation 1926 December 11.0 ET

\begin{tabular}{|c|c|c|c|c|}
\hline \multicolumn{2}{|c|}{$1913-1926$} & \multicolumn{3}{|c|}{$1926-1933$} \\
\hline$M_{0}=359.89405$ & \pm 0.00011 & $359: 89395$ & \pm 0.00011 & \\
\hline$\omega=171.75871$ & \pm 0.00080 & 171.75292 & \pm 0.00070 & \\
\hline$\Omega=196.24336$ & \pm 0.00050 & 196.24273 & $\pm 0.00050\}$ & 1950 \\
\hline$i=30.73808$ & \pm 0.00040 & 30.73870 & \pm 0.00040 & \\
\hline 0.7170158 & \pm 0.0000003 & 0.7170151 & \pm 0.0000004 & \\
\hline 3.5115892 & \pm 0.0000026 & 3.5116703 & \pm 0.0000025 & AU. \\
\hline
\end{tabular}

For the integration from 1913 back to 1900 we first adjusted the 1913-1926 elements by the mean of variations (d) and (c) and integrated to 1907 . We then corrected the 1907 elements similarly and continued the integration back to 1900 . The resulting $(\mathrm{O}-\mathrm{C})$ residuals obtained for 1900 ranged from 1.7 to $15^{\prime \prime}$. The 1900 and 1913 observations were then used to correct this orbit.

The two sets of elements for an epoch in 1913 are given below. The mean residuals were \pm 3.06 and \pm 2.42 , respectively, and the differences are listed in Table I as column (e). 


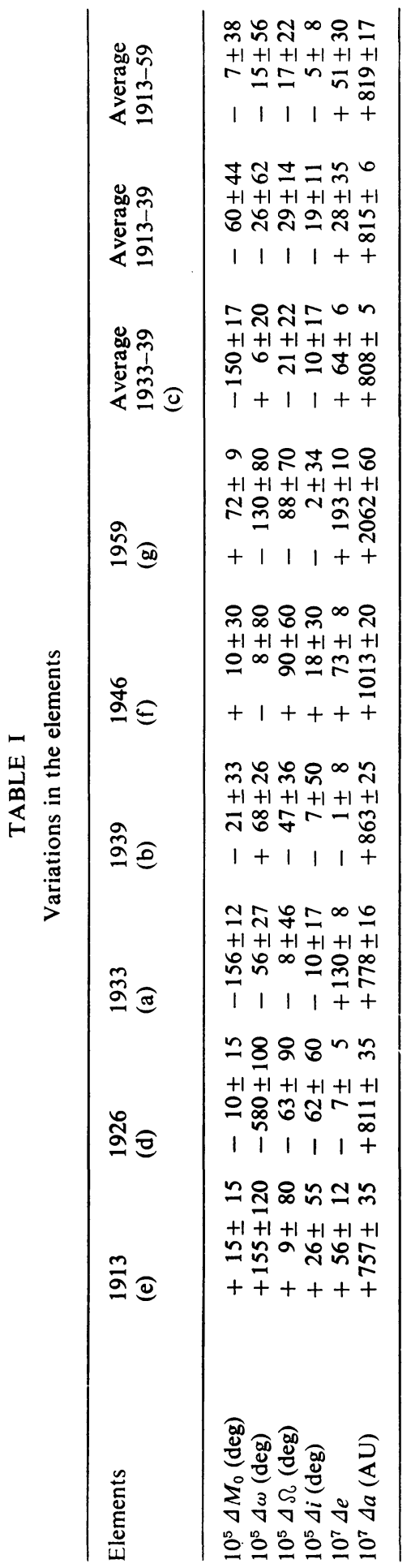


Epoch and osculation 1913 August 21.0 ET

\begin{tabular}{|c|c|c|c|c|}
\hline \multicolumn{2}{|c|}{$1900-1913$} & \multicolumn{3}{|c|}{$1913-1926$} \\
\hline$M_{0}=348.89657$ & \pm 0.00010 & $348: 89762$ & \pm 0.00011 & \\
\hline$\omega=171.48398$ & \pm 0.00090 & 171.48553 & \pm 0.00080 & \\
\hline$\Omega=196.36857$ & \pm 0.00060 & 196.36866 & $\pm 0.00050\}$ & 1950.0 \\
\hline$i=30.74613$ & \pm 0.00036 & 30.74639 & \pm 0.00040 & \\
\hline 0.7206350 & \pm 0.0000012 & 0.7206406 & \pm 0.0000003 & \\
\hline 3.4936561 & \pm 0.0000020 & 3.4937318 & \pm 0.0000030 & AU. \\
\hline
\end{tabular}

Linking the 1946 and 1959 apparitions was more difficult, probably due to the comet's approach to Jupiter in 1958. The final improved elements for 1946 are:

Epoch and osculation 1946 September 17.0 ET

\begin{tabular}{|c|c|c|c|c|}
\hline \multicolumn{2}{|c|}{$1939-1946$} & \multicolumn{3}{|c|}{$1946-1959$} \\
\hline$M_{0}=359.77761$ & \pm 0.00032 & $359: 77781$ & \pm 0.00080 & \\
\hline$\omega=171.81091$ & \pm 0.00025 & 171.81074 & \pm 0.00072 & \\
\hline$\Omega=196.29317$ & \pm 0.00020 & 196.29497 & $\pm 0.00060\}$ & 1950. \\
\hline$i=30.72683$ & \pm 0.00013 & 30.72720 & \pm 0.00030 & \\
\hline$e=0.7166747$ & \pm 0.0000003 & 0.7166894 & \pm 0.0000008 & \\
\hline 3.5143590 & \pm 0.0000019 & 3.5145616 & \pm 0.0000004 & $\mathrm{AU}$ \\
\hline
\end{tabular}

and on running the integration forward to 1965 we obtained the following improved sets of elements for 1959:

Epoch and osculation 1959 September 29.0 ET

\begin{tabular}{|c|c|c|c|c|}
\hline \multicolumn{2}{|c|}{$1946-1959$} & \multicolumn{3}{|c|}{$1959-1965$} \\
\hline$M_{0}=355^{\circ} .71004$ & \pm 0.00008 & $355: 71149$ & \pm 0.00004 & \\
\hline$\omega=172.84615$ & \pm 0.00072 & 172.84356 & \pm 0.00033 & \\
\hline$\Omega=196.03171$ & \pm 0.00060 & 196.02994 & \pm 0.00030 & 1950 \\
\hline$i=30.90435$ & \pm 0.00030 & 30.90440 & \pm 0.00016 & \\
\hline 0.7289063 & \pm 0.0000008 & 0.7289449 & \pm 0.0000006 & \\
\hline 3.4525903 & \pm 0.0000004 & 3.4530026 & \pm 0.0000060 & \\
\hline
\end{tabular}

The mean errors were $\pm 1 " .42$ and $\pm 5^{\prime \prime} .91$ in 1946 and \pm 5.90 and \pm 2 ".20 in 1959 . Since the comet made two revolutions between 1946 and 1959, the variations are halved, the results being given in Table I as columns (f) and (g).

The large residuals for 1946-1959 do not allow us to consider these elements as final, but they are suitable for the determination of the variation in the daily mean motion with fair reliability.

In the course of our analysis we obtained the following results: 
(1) Before being discovered in 1900 , the comet had rather a close approach to Jupiter. Our elements in 1900 were:

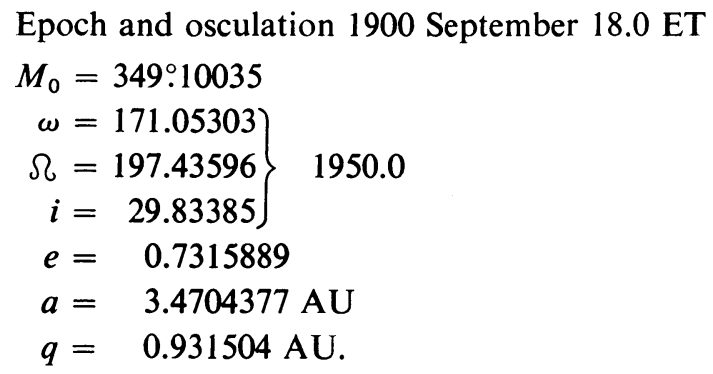

On 1898 October 27.92798 the comet was at its minimum distance of $0.1887 \mathrm{AU}$ from Jupiter. Some nine months earlier, when the comet had receded to $1.465 \mathrm{AU}$, its orbital elements were:

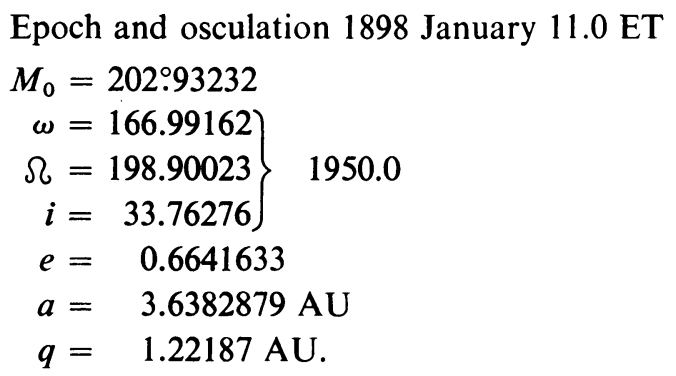

The decrease in perihelion distance from 1.22 to $0.93 \mathrm{AU}$ must have had a profound influence on the comet. On passing through perihelion in 1900 there would have been a significant change in the physical condition of the nucleus, and this apparently led to the ejection of meteoric particles.

From the variation of the elements, we deduce that the comet has a secular deceleration, amounting to about $0.081 \mathrm{day} /(\text { period) })^{2}$; this increases the comet's velocity, from which we conclude that most of the meteoric material was ejected back along the orbit. Having observed in 1933 and 1946 a rather dense swarm of meteoroids behind the comet, we can expect a denser swarm of such bodies ahead of the comet (or, at least, the more massive meteoroids; there is evidence of this in the large bolide observed at 53 locations on 1926 October 9).

(2) The comet approached Jupiter again on 1910 February 3.709, passing it at a minimum distance of $1.0049 \mathrm{AU}$. This encounter changed the orbit of the comet, bringing it closer to the orbit of the Earth $(q=0.976)$, and the subsequent cumulative action of planetary perturbations brought the orbits (at the comet's descending node) even closer. The uniform variation in the comet's daily mean motion indicates that ejection of meteors took place in a similar manner at every perihelion.

(3) The third approach to Jupiter during the period under investigation took 
place on 1958 January 19.5, the minimum distance being 0.93 AU. This approach caused the comet's perihelion distance to decrease to $0.936 \mathrm{AU}$. It is likely that this new approach brought about vigorous activity in the comet's nucleus and a further ejection of particles. The larger change in the elements (particularly the semimajor axis) from the 1946-1959 to 1959-1965 representations is consistent with this.

(4) Belyaev and the author have obtained predicted elements and an ephemeris for the 1972 return. This was based on 127 observations in 1959 and 1965. An approach of the comet to within $0.577 \mathrm{AU}$ of Jupiter on 1969 September 23 caused the perihelion distance to increase to $0.994 \mathrm{AU}$. This means that the orbits of the comet and the Earth will pass within $0.0006 \mathrm{AU}$ of each other, suggesting that there might be a return of the meteor showers, if not in 1972, then at the following returns of the comet. The Earth will pass near the descending node of the comet's orbit on October $8^{\mathrm{d}} 15^{\mathrm{h}} 45^{\mathrm{m}}$ UT. The comet will then be 58.5 days ahead of the Earth. This situation will be similar to that on 1933 October 9, the comet then passing the point of approach 80 days before the Earth.

The precise orbital elements we have obtained for $\mathrm{P} / \mathrm{Giacobini-Zinner}$ enable us to calculate orbits for Draconid meteors. Keeping in mind that the Earth passes the point of approach 58.5 days after the comet, let us imagine a meteor moving at the same distance from the comet, not in 1972 but in 1965, with the same orbital elements as the comet. Integration of the motion of this imaginary meteor gave an approach to Jupiter on 1969 August 16 (more than a month before the comet) to a distance of $0.710 \mathrm{AU}$. The resulting change in the meteor's orbit is very different from that in the comet's orbit, and the meteor will pass $0.0636 \mathrm{AU}$ from the Earth in 1972. On the strength of this calculation we suggest that all other meteors moving at approximately the same distance from the comet in 1965 will be diverted by Jupiter into very different orbits, and their subsequent encounters with the Earth will be very unlikely.

Observations in 1933 and 1946 indicated that meteoroids are ejected from the comet with velocities of the order of 13 to $14 \mathrm{~m} \mathrm{~s}^{-1}$. In order to encounter the Earth in October 1972 they must be 58.5 days behind the comet, which means that they should have made four revolutions around the Sun since leaving the comet. We have made calculations for four imaginary meteors ejected from the comet's nucleus near perihelion in 1946. We suppose that the meteors were ejected forward along the orbit of the comet at a velocity of $13.4 \mathrm{~m} \mathrm{~s}^{-1}$, when the comet's true anomaly was $+30^{\circ},+45^{\circ}, 0$ and $-30^{\circ}$, respectively. The subsequent elements of the orbits of the meteors are shown in Table II. It is clear that none of these meteors can encounter the Earth in 1972. We obtained a similar result when we traced up to 1972 the orbit of a meteor ejected in 1940. An intense shower like those of 1933 and 1946 is very unlikely in 1972. It is more likely that the shower will be poor or missing completely.

From these calculations we see that at every approach to Jupiter the meteor swarm is considerably dispersed (up to $0.1 \mathrm{AU}$ at perihelion for one approach). This supports our conclusions (Evdokimov, 1955) about the youth of the Draconid showers of 1933 and 1946. As further confirmation, we considered the ejection of meteors by the comet in 1940 with a moderate velocity $\left(13\right.$ to $\left.14 \mathrm{~m} \mathrm{~s}^{-1}\right)$ and calculated orbits for 
TABLE II

Orbital elements of meteors leaving the comet in 1946

\begin{tabular}{|c|c|c|c|c|c|c|}
\hline \multirow{2}{*}{$\begin{array}{l}\text { True ano- } \\
\text { maly of } \\
\text { meteor at } \\
\text { ejection }\end{array}$} & \multicolumn{3}{|l|}{1946} & \multicolumn{3}{|l|}{1959} \\
\hline & $a(\mathrm{AU})$ & $e$ & $q(\mathrm{AU})$ & $a(\mathrm{AU})$ & $e$ & $q(\mathrm{AU})$ \\
\hline$+30^{\circ}$ & 3.528562 & 0.717801 & 0.995757 & 3.455725 & 0.740067 & 0.898258 \\
\hline+45 & 3.527917 & 0.717730 & 0.995837 & 3.452743 & 0.750017 & 0.863128 \\
\hline 0 & 3.528465 & 0.717808 & 0.995706 & 3.456468 & 0.732909 & 0.923192 \\
\hline-30 & 3.528562 & 0.717801 & 0.995757 & 3.452074 & 0.740930 & 0.894330 \\
\hline \multirow{2}{*}{$\begin{array}{l}\text { True ano- } \\
\text { maly of } \\
\text { meteor at } \\
\text { ejection }\end{array}$} & \multicolumn{3}{|l|}{1965} & \multicolumn{3}{|l|}{1972} \\
\hline & $a(\mathrm{AU})$ & $e$ & $q(\mathrm{AU})$ & $a(\mathrm{AU})$ & $e$ & $q(\mathrm{AU})$ \\
\hline+30 & 3.452468 & 0.740449 & 0.896092 & 3.482198 & 0.728500 & 0.945415 \\
\hline+45 & 3.449419 & 0.750400 & 0.860976 & 3.477261 & 0.739697 & 0.905142 \\
\hline 0 & 3.453476 & 0.733301 & 0.921040 & 3.484520 & 0.720305 & 0.974460 \\
\hline-30 & 3.449106 & 0.741320 & 0.892216 & 3.478642 & 0.729328 & 0.941570 \\
\hline
\end{tabular}

several meteors ejected when the comet was at perihelion (1940 February 17). All the meteors with forward ejection velocities of $14 \mathrm{~m} \mathrm{~s}^{-1}$ would pass very close to the Earth $(0.001 \mathrm{AU})$ on 1946 October 10 around $3^{\mathrm{h}}-4^{\mathrm{h}} \mathrm{UT}$, i.e., at the maximum of the meteor shower. This is proof that the meteoroids which became the meteors in 1946 were ejected from the comet in 1940.

We were able to explain the 1933 meteor shower by a similar ejection from the comet in 1900. A meteor ejected forward from the comet in 1900 with a velocity of $15.0 \mathrm{~m} \mathrm{~s}^{-1}$ passed only $0.0004 \mathrm{AU}$ from the Earth on 1933 October 9. A meteor ejected at the same moment back along the orbit with a velocity of $14.5 \mathrm{~m} \mathrm{~s}^{-1}$ passed $0.02 \mathrm{AU}$ from the Earth in 1926, indicating that the meteor showers of both 1926 and 1933 were formed by meteoroids ejected in 1900 .

\section{Acknowledgments}

The calculations linking the apparitions of $1900,1913,1946$, and 1959 as well as all the calculations on imaginary meteors could be made only using the excellent computer programmes available now at the department of minor planets and comets of the Institute for Theoretical Astronomy. The programme by Belyaev (1972) was used for the integration - taking into account the perturbations by seven major planets - and the calculation of residuals. The programme by Bokhan (1972) was used for the orbit improvements. The author thanks N. A. Belyaev, N. A. Bokhan, and E. I. Kazimirchak-Polonskaya for the possibility of using these programmes and for suggestions made in the course of this investigation. My sincere thanks are due to the administra- 
tion of the Institute for Theoretical Astronomy for providing an opportunity for personnel from the Kazan State University to work at the Institute.

\section{References}

Belyaev, N. A.: 1972, this Symposium, p. 90.

Bokhan, N. A.: 1972, this Symposium, p. 86.

Dubyago, A. D.: 1956, Byull. Astron. Obs. V. P. Engel'gardta No. 32.

Evdokimov, Yu. V.: 1955, Astron. Tsirk. No. 159.

Evdokimov, Yu. V.: 1963, Astron. Zh. 40, 544.

\section{Discussion}

L. A. Katasev: What is the basis for your statement that a great cluster of large meteoroids moves ahead of the nucleus of $\mathrm{P} / \mathrm{Giacobini-Zinner}$ ?

$Y u$. V. Evdokimov: I omitted to say at the beginning of my report that I agree with the widely approved model that a comet is a conglomerate of ices and solid particles. My work is based on the fact that the particles are liberated when the comet comes close to the Sun. Since this comet has a secular deceleration most of the meteoric material is ejected back from the nucleus and moves ahead of the comet.

S. K. Vsekhsvyatskij: Have you made an attempt to estimate the mass of the comet's nucleus through consideration of the nongravitational forces and from the data on the Draconid meteor shower?

$Y u$. V. Evdokimov: I have determined a rate of mass loss, rather than the total mass of the comet's nucleus. In 1963 I showed that the mass loss is as great as about $10 \%$ per revolution. I came to this conclusion from the displacement of the orbital planes of the meteors from that of the comet. Using the programme by Belyaev, I have now been able to determine the displacement of the orbital planes of meteoroids ejected in 1940 and 1946 . I now find that the comet ejects about $5 \%$ of its mass per revolution, so that it will cease to exist after 50 to 100 revolutions. 